\title{
ARTICLE
}

\section{Practical assessment and management of risk in sexual offenders ${ }^{\dagger}$}

\author{
Katharine Russell \& Rajan Darjee
}

\begin{abstract}
Katharine Russell is a consultant forensic clinical psychologist and Rajan Darjee is a consultant forensic psychiatrist. They co-lead the NHS Lothian Sex Offender Liaison Service, which offers consultation, assessment and treatment to help criminal justice and other agencies manage complex sexual offenders in the community. Their clinical and research interests are paraphilias and personality disorders in sex offenders, and the implications of these for risk management in the community. Correspondence Dr Rajan Darjee, NHS Lothian Sex Offender Liaison Service (SOLS), The Orchard Clinic, Royal Edinburgh Hospital, Morningside Terrace, Edinburgh EH10 5HF, UK. Email: rajan.darjee@ nhslothian.scot.nhs.uk
\end{abstract}

${ }^{\dagger}$ For a companion article in Advances, see Darjee R, Russell K (2012) What clinicians need to know before assessing risk in sexual offenders, 18: 467-78.

\begin{abstract}
SUMMARY
This article focuses on how to assess and manage risk in sexual offenders. It covers assessment issues, including interviews, taking a sexual history, assessing sexual deviance and assessing personality. The key step of case formulation is outlined and the development of risk management strategies, including monitoring, supervision and treatment, is described.

\section{DECLARATION OF INTEREST}

None.
\end{abstract}

In our previous article on assessing and managing risk in sexual offenders (Darjee 2012), we covered the issues clinicians need to be aware of before assessing risk. In the current article, we will present a pragmatic comprehensive approach to assessing risk and planning risk management. We will set out an approach based on the structured professional judgement approach to risk assessment. This approach is particularly useful for mentally disordered, complex or unusual sexual offenders, and provides a framework not only for assessing risk, but also for planning risk management. Actuarial and dynamic risk instruments, as well as specific structured professional judgment tools, can be used within the approach we present here, as set out below.

As in our previous article, we will focus on adult male offenders. The broad structure of the approach suggested here would be appropriate for females and young people too, but the specific factors to look at, the instruments to use, and treatment and management approaches need to be informed by the relevant literature and clinical guidance. Risk assessment tools for males should not be used with females, and assessment and management of female sexual offenders should be informed by the emerging literature (Gannon 2010). There are specific risk assessment instruments for adolescents, for whom assessment needs to take a developmental perspective, and treatment and management may need to have a more systemic focus (Rich 2009).

Psychiatrists and other mental health professionals need to consider risk of sexual offending in a number of mental health and criminal justice contexts. When preparing reports for criminal courts, assessment of risk of sexual violence contributes to decisions about whether:

- an individual is remanded in custody or placed on bail

- a mentally disordered offender is placed in a secure hospital or on a restriction order

- a community or custodial sentence is imposed

- in serious cases, an extended sentence or indeterminate sentence is imposed.

Risk is crucial in the use of mental health legislation, and in some cases sexual violence will be of relevance. Risk of sexual offending is relevant for a significant minority of patients in secure and other in-patient settings and when considering community management. Through Multi-Agency Public Protection Arrangements (MAPPA), psychiatrists, psychologists and other mental health professionals increasingly contribute to the management of sexual offenders on community sentences or on release from prison. Those who sit on, or produce reports for, parole boards need to consider risk of sexual offending and community management.

Risk of sexual offending arises in child protection and adult protection contexts. Professional bodies (who regulate doctors, teachers, the clergy, social workers or other professionals) may seek assessments where there has been behaviour of concern. There may also be involvement in cases where certain civil orders are being considered by the police (e.g. sexual offences prevention orders, risk of sexual harm orders, foreign travel orders), and when considering whether a sexual offender should remain subject to notification requirements.

\section{Assessment}

The aim of assessment is to gather sufficient information to determine the presence of risk and 
protective factors and to formulate an understanding of a case so as to inform risk management. Assessment involves gathering information from interviews, documents and tests. Practitioners should obtain information from a range of sources in the multiple agencies covering different areas of functioning.

\section{Interviews}

The first task in an interview is to engage the person. You may have to spend some time at the beginning explaining what you are doing and allaying concerns. Individuals may be embarrassed, reticent, suspicious or wary. When we assess sex offenders we undertake at least two interviews, each lasting $2 \mathrm{~h}$. This allows us to see how a person presents at different times and to postpone asking about more difficult areas (such as sexual history and offending history) until some rapport and trust has been established. We use two interviewers, one male and one female. This allows for assessment of attitudes towards, and ability to relate to, each gender, allows one person to observe while the other interviews, provides for a consensus rating of personality and risk tools, and allows for some mutual support and supervision. There may be concerns that having two interviewers will affect the power dynamic, placing the interviewee at a disadvantage and hampering rapport building; in practice, we have not found this to be a problem. The standard areas of the psychiatric history should be covered but time needs to be spent on personal history, relationships and sexual history, personality, offending history (sexual and non-sexual, allegations as well as convicted offences), and response to interventions (in the community and in hospital or prison). As well as the traditional mental state examination, there is a need to look at current understanding of interpersonal, emotional and sexual functioning; understanding of offending and risk; attitude towards future interventions; and future plans.

\section{Taking a sexual history}

It is important to assess sexual development, knowledge, interest, relationships and functioning. Many psychiatry and clinical psychology trainees who work with us feel unsure of and uncomfortable with this. Being able to engage in a discussion about sexual matters is important. The interviewer must have some knowledge of 'normal' sexual development and functioning, and confidence to ask about sexual interests and behaviour. This part of the assessment should be conducted in a comfortable, confident and matterof-fact manner, which is sensitive to, and tries to alleviate, the potential embarrassment of the interviewee, so they feel comfortable enough to discuss these intimate issues. Box 1 lists the areas that the assessment should cover.

Reluctance and reticence when doing this part of the assessment probably arise from lack of specific training, discomfort talking about sex and doubts about the validity of the answers. If the person is put at ease and given positive feedback for speaking openly about sexual matters, it should be possible to elicit reliable information about sexual issues. Nevertheless, given the importance of deviant sexual interests as a risk factor, more objective approaches may be required too.

\section{Assessment of sexual deviance}

There are inherent difficulties associated with self-report of any behaviour, and this may be particularly problematic with sexual behaviour. However, 'objective' methods for assessing sexual deviance can be applied to mitigate this problem.

\section{Penile plethysmography}

Penile plethysmography involves the monitoring of the participant's penile responses (often along with other psychophysiological responses) while

\section{BOX 1 Sexual history}

- Sexual knowledge and sources of information

- Sexual attitudes to self and others (including attitudes permissive of sexual behaviour with children, rape supportive beliefs and general attitudes to sex), sexual self-esteem and sexual narcissism

- Sexual development: age at onset and development of puberty, sexual interests, masturbation, dating, sexual intercourse

- Relationships history: age of self and partners, gender of partners, duration, number of relationships (including number of age-appropriate sexual partners), quality of relationships, problems, fidelity and abuse

- Fantasy: content, use and development

- Orientation: sexual interest in males, females, children

- Sex drive: strength of libido, sexual preoccupation (including frequency of sexual outlets over a period of time), hypersexual arousal

- Sexual dysfunction: impotence, premature ejaculation, delayed ejaculation

- Experience: range of sexual behaviours and specific questions about paraphilias

- Current sexual practices: mood, material used, thoughts or visual images, conditions for arousal during sex and masturbation, frequency and nature of sexual outlets

(Adapted from Brockman \& Bluglass 1997) 
he views or listens to material depicting sexual behaviour (e.g. consenting sex, coerced sex, sex involving gratuitous violence) or individuals who may or may not be sexually arousing (e.g. children, teenagers, adults) (Federoff 2009). Penile plethysmography requires a dedicated laboratory and qualified staff to carry out the assessment. It is used to look at patterns of sexual arousal, to assist in treatment and risk management planning, and to assess change with treatment. It cannot be used to determine whether someone has committed a specific offence or as a risk assessment in and of itself. In England and Wales, penile plethysmography is available in some highsecurity hospitals and some prisons, but it is not available in any setting in Scotland.

Penile plethysmography remains controversial. Different sites use different stimuli and different assessment methods, and the need for consistency of approach has been highlighted. Questions arise over whether individuals can 'fake good' on the test and assessors must try to control for this. It has been argued that there is limited evidence for the utility of penile plethysmography, but in complex cases, particularly where the issue of response of sexual deviance to treatment arises, it can add another piece of information that helps with assessment. Given the evidence base for penile plethysmography assessed sexual deviance (particularly sexual interest in children) as an important risk factor for sexual recidivism (Hanson 2005), it is difficult to justify not having access to this assessment method (as is currently the case in the jurisdiction in which we work).

\section{Viewing-time assessment}

Viewing-time measures are based on the finding that people spend longer looking at images that are sexually appealing. Computer programs such as Affinity 2.5 (Glasgow 2009) and the Abel Screen (Abel 2009) can provide this assessment by presenting images and measuring how long a person looks at them, perhaps while undertaking a task. The viewing-time test may be combined with a questionnaire asking about how sexually arousing the images are and/or about sexual interests more generally. A promising further development is the Explicit and Implicit Sexual Interest Profile (EISIP; Banse 2010), combining self-report, implicit association tests and viewingtime measures. Studies have shown that viewingtime measures can distinguish between deviant and non-deviant offenders, sometimes better than penile plethysmography can (Letourneau 2002). Viewing-time measures are primarily used to assess sexual interest in children.

\section{Objective offending behaviour data}

Two scales have been developed: one to assess paedophilia (Screening Scale for Pedophilic Interests, SSPI; Seto 2008) and the other to assess sexual sadism (Sexual Sadism Scale, SSS; Marshall 2006a). Both are based on objective behaviour reported in documents such as victim statements and police reports, not self-report.

The SSPI has 4 items which are weighted and summed to give a total score out of 5 . A score of 4 or 5 indicates a strong likelihood that there is a greater sexual interest in children than in adults, based on studies comparing the SSPI with penile plethysmography assessment; high scores are also associated with recidivsim.

The SSS is a checklist of behaviours associated with sadism. The authors created the scale after finding that literature definitions of sadism varied and diagnostic reliability and validity using criteria such as those in DSM-IV were lacking (Kingston 2008). They sought to create a dimensional structured assessment based on what the literature reported to be behavioural indicators of sadism (e.g. torture, abduction, strangulation). There are clear definitions for each behaviour and items are rated on a 3-point scale. Items are weighted and summed to yield a total score, and there are two cut-offs: one for definite and one for possible sadism. The scale is relatively new and has been validated in only one study (Nitschke 2009), but provides a useful framework for structuring consideration of sexual sadism.

\section{Self-report measures}

A number of self-report measures are available to assess sexual interests, knowledge and functioning (e.g. Multiphasic Sex Inventory, MSI; Nichols 1984). Some are very long and off-putting. They can be quite transparent and should never be the sole assessment method used. We do not routinely use these measures as we have found they add little to the understanding of a case.

\section{Assessment of personality}

Sex offenders have significant rates of personality dysfunction and overt personality disorder (see Darjee 2012). Therefore personality and personality disorder should always be considered, particularly in serial, serious harm, unusual or difficult offenders. An assessment of personality disorder should involve a structured assessment such as the International Personality Disorder Examination (IPDE; Loranger 1999). Given the literature on psychopathy and risk, an assessment of psychopathy using the Psychopathy ChecklistRevised (PCL-R; Hare 2004) or Psychopathy 
Checklist: Screening Version (PCL:SV; Hart 1995) should also be undertaken. We incorporate questions from the IPDE and PCL-R into our assessments. When completing an assessment of personality, self-report measures can be helpful (e.g. Millon Clinical Multiaxial Inventory-III, MCMI-III; Millon 1997). However, they should never be used as a sole means of assessing personality, owing to self-report bias.

When assessing personality in sexual offenders, we are less interested in cut-off scores and diagnostic categories than in the range of traits and areas of personality dysfunction an individual has. We are seeking to understand and formulate the person's interpersonal, emotional and self-functioning. Personality is multilayered and dimensional, not categorical. With DSM-5, radical changes are being proposed to the classification and diagnosis of personality disorder. These include having fewer diagnoses and taking a more dimensional approach to assessment (Widiger 2011).

\section{Psychometric tests}

A range of self-report psychometric tests can be used with sex offenders (Craig 2009). Many are completed pre- and post-treatment in sex offender treatment programmes. These include measures of self-esteem, impulsivity, locus of control, anger, attitudes, sexual interests, personality and anxiety. They are transparent and prone to self-report bias. There is conflicting evidence on the relationship between self-report psychometrics and recidivism, with a few studies finding a positive association (e.g. Beggs 2011). Changes in scores may or may not indicate a reduction in risk, but there is little reliable evidence that such measures indicate whether there has been positive change with treatment or whether risk has reduced. They may be helpful in individual case formulation but rarely tell you anything you did not already know from a comprehensive assessment, including assessment of personality and risk as outlined here. There is little to recommend them when assessing risk.

\section{Which risk assessment instrument?}

We described and discussed risk assessment instruments in our previous article (Darjee 2012). An appropriate sexual offending risk assessment instrument should be used to identify and combine risk factors. We use an actuarial tool (Risk Matrix 2000; Thornton 2010) and a structured professional judgement tool (Risk of Sexual Violence Protocol, RSVP; Hart 2003). Criminal justice agencies we work with (police, criminal justice social work and prison) use Risk Matrix 2000 and a dynamic tool (Stable and Acute 2007; Hanson 2007). When we consult to these agencies, the factors identified by these tools and a cautious interpretation of total scores inform the case formulation (as outlined below). The individuals we directly assess have a mental disorder (usually personality disorder), are complex and /or unusual, so we use the RSVP. As the RSVP is now almost 10 years old, we also take into account the more recent evidence base when considering risk factors. We are cautious about the relevance to future risk of denial and minimisation. On the other hand, we are concerned about sexual preoccupation, use of sex as a coping mechanism, and personality disorder; and we explicitly consider hostility towards women, sexual entitlement and grievance thinking when we look at attitudes. The Structured Assessment of Protective Factors (SAPROF; de Vogel 2009) can be used to structure the consideration of protective factors, alongside instruments focused on risk factors.

As most reoffending by sexual offenders is non-sexual, it is important to consider whether instruments for other types of risk should be considered, for example non-sexual violence, domestic violence, stalking or general offending. Where there is a history of non-sexual violence, violence in sexual offending and, in men, attacks on adult females, we use the Historical, Clinical, Risk Management-20 tool (HCR-20; Webster 1997). Where the victim of a sexual offence has been a partner or a partner's child, or there is a history of domestic abuse, we use the Spousal Assault Risk Assessment (SARA; Kropp 1995).

\section{Putting it all together: an approach to case formulation}

The most important part of the assessment is not the tool used, but the interpretation of the factors identified through a case formulation (Hart 2011). An assessment is more than choosing which tools are appropriate and completing them. The tools merely allow for the case to be examined against known factors and dysfunctional personality traits. A clinician must then formulate the case to explain which factors have been pertinent in this person's pathway to offending and relevant to understanding the way they have offended, who they have offended against and why they have offended. Organising a structured formulation around predisposing, precipitating, perpetuating and protective factors provides a useful framework.

We use the following seven steps when formulating a case.

1 Summarise the risk and identified protective factors. Organise the risk factors into static, stable dynamic and acute dynamic factors. 
Organise the protective factors into intrinsic strengths and extrinsic supports/controls.

2 Briefly consider the aetiology of dispositional factors (i.e. stable dynamic risk factors and intrinsic strengths) looking at childhood, adolescent and adult development. Early family dynamics and attachment, and the negotiation of emerging sexuality in childhood and adolescence are very often important, particularly in personality disordered and/ or sexually deviant offenders. How have dispositional factors changed over time (perhaps with intervention)? What are the key underlying dispositions in the case? How do the dispositional factors interact with each other and account for historical/static factors?

3 Consider the role of mental disorders. Do personality disorder, intellectual disability, chronic mental illness or paraphilia underpin stable dynamic factors? What role have mood disorders, psychosis or substance misuse played in precipitating offending? How will any mental disorder affect the ability and willingness of the person to engage with and respond to interventions?

4 Analyse the offending behaviour, looking at: (a) the pattern of offending over time; (b) types of victims and why they were selected; (c) proximal precipitants/disinhibitors/destabilisers/ triggers; (d) motivation (what needs was the person fulfilling through offending, perhaps by considering typology); (e) how did the person offend (approach to victim, control of victim, preventing detection); (f) where there are multiple offences, if, how and why has this changed over time.

5 Look at periods of non-offending to identify factors that may be protective or which may indicate the person is moving towards a more prosocial life. Consider factors from the desistance literature (Laws 2011) and look at how the person was achieving 'primary goods' (see below) in non-antisocial ways (Ward 2006).

6 Examine response to previous interventions and management approaches in institutions and the community. If they worked, why did they work? If not, why not? Were interventions delivered in such a way as to be responsive to the individual?

7 Do other issues need to be understood? The most common 'other issue' in our cases is the relational dynamics between the individual and staff, particularly where sex offenders have personality disorders. This often mirrors early family dynamics and the dynamics of the person's offending.
We then set out narrative risk scenarios. Using the formulation, we generate scenarios that lay out the plausible ways in which the offender may reoffend in the future. These scenarios must be anchored in the risk and protective factors, the case formulation and knowledge of the literature on sexual offending. A wide range of scenarios should be considered initially (e.g. best-case, worst-case, escalation, repeat and twist) and then 'pruned' so that only the plausible scenarios are considered in detail and used to generate a risk management plan that is proportionate to the risk (Hart 2011).

When we present the conclusions of our assessments we avoid terms such as low, medium and high risk. Narrative descriptions of future risk scenarios laying out what the person has the potential to do, to whom, under what circumstances are of more pragmatic use. Labels such as high, medium or low risk are meaningless unless there is a clear definition so that they communicate some meaning. We never quote percentage reoffending rates from actuarial instruments in our assessments, because specific rates quoted for tools are from cohorts in another geographical location or time, so it is unlikely that the specific percentages will apply to our cases.

Although labels such as high, medium and low may not be particularly useful in managing an individual case, there is a need to prioritise resources towards the management of higherrisk cases. For example, under MAPPA (see below), when considering prioritisation of cases for treatment programmes and when considering some legal orders (e.g. indeterminate sentencing), an allocated risk level will be necessary. When using such levels it is important: to have clear criteria; to know which criteria are being used in a particular decision in a particular case; and to be aware that different criteria focusing on different aspects of risk may lead to different risk level conclusions in the same case. For example, we have come across cases which are 'low risk' on assessment with an actuarial tool but 'high risk' using MAPPA definitions and 'medium risk' using the criteria for indeterminate sentencing in Scotland (Darjee 2011).

\section{Management}

The aim of risk management is to reduce the likelihood and impact of harm to others by developing and enhancing protective factors (both extrinsic supports and intrinsic strengths). This can be achieved through interventions to ameliorate key stable dynamic factors (i.e. changing underlying dispositions towards offending), restricting the 
opportunity for reoffending, and monitoring for acute factors (triggers, situations and/or states) which indicate that an offence may be imminent. It is important that the risk management plan is linked to the risk assessment, and that an individualised and proportionate approach is adopted. A risk assessment is only of use if it informs and aids risk management, and a risk management plan is only of use if it is based on an individualised and evidence-based assessment. If a risk assessment has been completed using the approach advocated in this article, concluding with a formulation and narrative risk scenarios, then risk management strategies should flow from the assessment in a fairly straightforward way. Risk is dynamic, changing over time, so it is important that the management of a case is reviewed and adjusted in the light of such changes.

In too many cases risk management is generic, rather than individualised, based on a risk level (high, medium or low), an offender type (paedophile or rapist), or defensiveness generated by organisational or staff anxiety. When constructing a risk management plan it is important to prioritise and put in place the necessary key measures. This is particularly important in complex high-risk cases in which there may be an overwhelming number of risk factors, few (if any) protective factors, a complex formulation and where future scenarios point to a dreadful outcome. A common mistake in such cases is to put in place far too many risk management strategies. What are the crucial things to monitor, what is absolutely necessary in terms of restrictions, what are the treatment interventions that are likely to lead to gains, and do particular potential victims need to be protected?

\section{Monitoring}

Monitoring (Box 2) involves repeated assessment of acute dynamic, stable dynamic and protective factors and compliance with restrictive measures.

\section{BOX 2 Key monitoring techniques}

- Talking to the offender

- Talking to people who know the offender

- Overt or covert surveillance

- Use of closed-circuit television

- Urine drug testing

- Alcohol breath testing

- Polygraph interviews

- Checking the offender's use of media/internet

- Checking the offender's associations with others
Considering the risk scenarios in a case can help in deciding whether things are going in the right direction. If an individual is in an institution (hospital or prison) it can be useful to monitor 'offence paralleling behaviours'. These are behaviours that are functionally similar in terms of their antecedents, meaning and function to an individual's offending behaviour (Daffern 2010). Polygraph interviews are controversial, but there is evidence that they can enhance community management and there is legislation permitting their mandatory use in England and Wales (Grubin 2007).

When monitoring an individual, particularly in the community, it is important that it is clear who will be monitoring what, when and how, and what will happen if there is cause for concern. Offenders and their families should be actively involved and engaged in the monitoring process where possible.

\section{Supervision}

Supervision involves placing restrictions on the offender (Box 3). The type and level of restrictions required will depend on the risk scenarios, the ability to self-manage and the level of external support. Overly restrictive supervision may be counterproductive, and in some cases may increase the risk posed by the individual. 'One size fits all' approaches should not be used. For example, although some men who have committed sexual offences against children should not be allowed contact with any child, this should not automatically be a recommendation with all child sex offenders. A long list of restrictions may be more about dealing with staff or organisational anxiety than about decreasing the likelihood of offending.

\section{BOX 3 Examples of supervision strategies for sex offenders}

- Electronic monitoring
- Exclusion zones
- Geographical restrictions
- Preventing contact with potential victims
- Notification to others of whereabouts and other
personal details
- Preventing contact with other sex offenders or previous
victims
- Preventing use of electronic equipment
- Detention in institutions
- Limiting housing options
- Mandatory assessment at day centres
- Limiting alcohol or drug use

- Electronic monitoring

- Exclusion zones

- Geographical restrictions

- Preventing contact with potential victims personal details

- Preventing contact with other sex offenders or previous victims

- Preventing use of electronic equipment

- Detention in institutions

- Limiting alcohol or drug use 
Where there are too many restrictions, inevitably they will not be applied consistently, and offenders and staff often lose track of them. Having lots of restrictions can be particularly counterproductive where offenders have narcissistic, paranoid or antisocial personalities.

The ultimate restriction that can be imposed is detention in an institution (e.g. prison or a secure hospital). But even in institutions there may be a sexual risk to staff or other prisoners/patients, so some restrictions may be necessary. Although some sexual offenders will abide by voluntarily imposed restrictions, a legal framework (such as probation, parole, notification requirements, mental health legislation or civil prevention order) is required to impose such restrictions.

Electronic tags are used to monitor some sex offenders in the community, usually to ensure that a curfew is being adhered to. There is limited evidence that such tagging reduces offending (Button 2009). In the USA, sex offenders are denied access to certain areas, so-called exclusion zones (Council of State Governments 2010). The confluence of exclusion zones in neighbouring areas may mean the only places they can live are in swamps and under freeways. Such restrictions can lead to instability and limited access to employment, Social Services and social support (Levenson 2007), can lead to sex offenders going missing, and may ultimately increase the risk of recidivism.

Some states in the USA have adopted community notification, with websites giving details of sex offenders living in an area. Mobile telephone applications and three-dimensional computer maps are available to the public. In the UK there is no such public notification, but disclosure to certain individuals (e.g. employers, accommodation providers, schools) may occur if this is merited by the assessment of risk in an individual case. A trial scheme to allow parents to seek information from the police regarding individuals they are concerned about (e.g. a new partner who has access to children) has been extended across Scotland. However, most new sexual offences are not committed by individuals with a conviction for sexual offending.

Blanket notification (like restriction zones) can alienate sexual offenders and even increase risk. Although blanket or limited notification may reassure the public, there is no evidence that it contributes to reducing the risk of sexual violence. Best practice would suggest that any notifications or disclosures should be reserved for the most risky cases based on an individualised assessment where other risk management strategies are insufficient.
BOX 4 Specific treatments for sexual offenders

Psychological

- Group cognitive-behavioural therapy (CBT)

- The Risk, Needs, Responsivity approach (Andrews 2007)

- The Good Lives model (Ward 2006)

Pharmacological

- Antilibidinals (e.g. gonadotrophin-releasing hormone agonists, cyproterone acetate

- Selective serotonin reuptake inhibitors (SSRIs)

\section{Treatment}

Treatment includes all interventions which aim to improve psychosocial functioning by alleviating symptoms, changing problematic areas of functioning and improving skills which will allow more prosocial functioning. Treatment aims to ameliorate stable dynamic factors, develop intrinsic strengths, increase extrinsic supports, prevent the occurrence of acute states or circumstances, and promote prosocial self-management. Although specific treatment programmes and medications (such as antilibidinals) are important in the treatment of sexual offenders (Box 4), it is important to think of treatment more broadly. Other mental health interventions (psychological treatments, occupational therapy, medication for psychiatric disorder), the development of working relationships with staff, the mustering of personal support and broader rehabilitative measures (e.g. help to gain employment) can all play a role. Here we will give an overview of specific treatment approaches for sexual offenders.

\section{Psychological treatment}

Psychological treatment for sexual offenders was primarily psychodynamic until the 1960s, when behavioural treatments were introduced (behavioural modification of sexual deviance and social skills training). Then treatment became cognitive-behavioural, incorporating work to address denial/minimisation, victim empathy, dysfunctional attitudes and relapse prevention (borrowed from substance misuse treatment). More recently, there has been emphasis on evidence-based dynamic factors (such as sexual deviance, poor coping, impulsivity, intimacy deficits), responding to individual needs and a more positive goal-oriented approach, with fewer punitive, confrontational and confessional aspects. 
In current practice, treatments should aim to:

1 help the individual understand the factors that are of relevance to their risk of sexual offending by developing a self-formulation;

2 give the individual the opportunity to address these factors by helping to make changes and develop skills;

3 help the individual make plans for the future, so they can lead their lives and meet their needs prosocially without causing sexual harm to others.

A number of aims that are assumed to be important but are of no real significance include getting the person to admit to their offence, to admit they are a 'sex offender', to show remorse, to express empathy for their victim, and to display 'good behaviour' in a group setting. As with most psychological interventions, the process of therapy is probably more important than the specific tasks or modules (Marshall 2006b).

Group-based cognitive-behavioural therapy (group CBT) for sex offending is regarded as the treatment of choice (Hanson, 2009). In the UK, these programmes are available in prisons, some secure hospitals, and through probation in the community. At the time of writing, sex offender programmes in prison and in the community in England \& Wales and in Scotland are being reviewed and updated, to take into account recent international evidence and practice. Meta-analyses point to the effectiveness of these interventions at reducing reoffending by about $40 \%$ (e.g. Hanson 2009), although some experts still doubt whether there is evidence that treatment is effective (Rice 2003).

There is evidence (Hanson 2009) that sex offender treatment is more effective when it follows the Risk, Needs, Responsivity approach known to be successful in general offending behaviour programmes (Andrews 2007). That is, treatment should be delivered to more rather than less risky individuals (Risk), should target factors (criminogenic needs or stable dynamic factors) associated with recidivism (Need) and should be delivered in a way matched to the learning style of individuals (Responsivity).

The Good Lives model (Ward 2006) has been increasingly influential on sex offender treatment. It proposes that sexual offenders share the same inclinations and basic needs as other people, and are naturally predisposed to seek certain goals, or 'primary goods'. The model encourages us to see sex offenders as people who want the same things in life as everyone else (e.g. relationships, knowledge, community, inner peace), but who have attempted to acquire such goods by offending or because they lack the skills to obtain them in non-offending ways. Therefore, treatment should focus on helping the person achieve their needs and goals in prosocial ways. The Good Lives model is not a treatment as such, but a philosophy to guide treatment. We find it a useful framework not just for treatment but for wider aspects of risk management, helping to focus not just on risk and restrictions, but on achieving positive prosocial outcomes (which reduces risk).

As most sexual offenders with mental disorder have similar psychosexual problems to those without mental disorder, and risk factors are the same in both groups, psychological treatment to address sexual offending should be available for higher-risk sexual offenders with mental disorders. Programmes are available in high and some medium secure units. There is no specific research on programmes for mentally ill sex offenders but programmes for sexual offenders with intellectual disability have been evaluated (Craig 2010). Before patients take part in these programmes, their mental states should be stable and the programmes should be adapted to their interpersonal, cognitive, emotional and perceptual deficits.

Many of the areas targeted in treatment (i.e. stable dynamic dispositions) relate to dysfunctional personality traits and many individuals who go through these programmes in criminal justice settings have personality disorders. Adaptations for individuals with high levels of personality dysfunction and psychopathy have been made (Dowsett 2008), including: greater focus on motivation, engagement, maintaining participation; using more appropriate learning styles; greater flexibility; emphasis on individual formulation and positive psychology; and incorporating specific elements of personality disorder therapies (e.g. schema, mentalisationbased or cognitive analytic therapy). Whether such approaches for sex offenders reduce recidivism awaits evaluation.

The 'accepted wisdom' that treatment makes psychopaths worse is not supported by more recent research (Barbaree 2006), and some treatment programmes have reported reductions in offending by psychopathic offenders similar to those achieved by non-psychopathic offenders (Skeem 2009), albeit that the base rate of reoffending in this group is higher. Some offending behaviour programmes exclude individuals with psychopathy, as they are considered unresponsive and disruptive. Some programmes accept individuals with psychopathy, but limit groups to no more than one or two 
such individuals. Other programmes have been developed, for example in England and North America, primarily for offenders with severe personality disorders, including psychopathy.

Whichever programme is followed, the most important issue is having the right approach, in terms of the competence, qualifications, experience, supervision and training of staff; the way treatment is delivered; proper resourcing and support for any treatment programme; and integration of treatment with other aspects of risk management.

\section{Medication}

Antilibidinals and SSRIs may be prescribed alongside psychological interventions to treat individuals who have problems with sexual regulation (sexual preoccupation, sex as coping or sexual deviation) that are not adequately addressed by other interventions (Grubin, 2008). Psychiatrists have an important role to play in assessing and treating sexual offenders who may benefit from medication, in prison and in the community. Medication should be given on a voluntary basis and the decision to prescribe should be based on the individual's clinical presentation.

Selective serotonin reuptake inhibitors may be more beneficial where there are intrusive fantasies or urges or an element of obsession, or where sexual fantasy or behaviour is associated with low mood or anxiety. Antilibidinals are more appropriate where there is hypersexual arousal or deviant sexual urges which are subjectively difficult to control. Newer gonadotrophin-releasing hormone agonists are potent antilibidinals, which can be given by long-acting injection and may have less troubling side-effects than drugs such as cyproterone acetate (Briken 2003).

Guidelines and protocols are available on the referral and treatment of sex offenders in prison or on probation in England \& Wales and in Scotland.

\section{Victim safety planning}

Victim safety planning may involve restricting access to specific named victims or a group of potential victims (e.g. children). Disclosure to schools, families, partners, employers and staff can also be used to minimise the risk that an offender may pose. The decision to disclose should be carefully considered in each case and based on an individualised risk assessment.

\section{MAPPA}

In the UK, MAPPA provisions (Kemshall 2009) have been introduced through legislation in England \& Wales and Scotland. Agencies involved in managing sexual offenders (police, probation/criminal justice social work, prisons and health service) are required to work together to put in place multi-agency arrangements for risk management. Depending on the level of risk posed and the complexity of multi-agency risk management, a case is allocated to one of three levels of risk management.

Risk assessment and management is central to the MAPPA process. The MAPPA framework is primarily about ensuring good communication and multi-agency working, especially where sexual offenders are assessed as posing a risk of serious harm to others. It can provide a valuable framework for clinical input for sexual offenders with personality disorders and paraphilias within a primarily criminal justice context (Russell 2012). In such cases, clinicians can focus on assessment, consultation and treatment, whereas other agencies deliver other aspects of risk management (supervision, monitoring, support, victim safety planning).

\section{Circles of Support and Accountability}

Circles of Support and Accountability was established in Canada to provide social support and monitoring for very-high risk sexual offenders released at the end of a prison sentence with no statutory community supervision. A group of trained and supported volunteers provides a network of support for the individual, helping the person to establish themselves in the community, to achieve goals in prosocial ways and to manage the risk they pose. There is evidence that this approach reduces recidivism. Similar projects have been established in parts of the UK (Harvey 2011).

\section{Conclusions}

Whether sexual offenders have a mental disorder or not, they should undergo an assessment of risk using appropriate instruments that cover evidencebased risk factors. Assessment involves gathering sufficient information from interviews, third parties and records, so that risk and protective factors can be identified. In complex cases it is important to undertake a thorough assessment of personality disorder and sexual deviance. The clinician should aim to produce a formulation which ties together the relevant risk and protective factors, helps in understanding why the person has offended in the way that they have, and looks to the future by setting out narrative risk scenarios. Risk management plans should flow from the risk formulation and scenarios, and should include monitoring, supervision, treatment and victim 
safety planning. Risk management should be proportionate and tailored to the individual case. Unnecessary restrictions should be avoided. Using an evidence-based approach grounded in a proper understanding of the individual can help guard against an approach distorted by subjective anxiety and fear.

\section{References}

Abel GG, Wiegal M (2009) Visual reaction time: development, theory, empirical evidence, and beyond. In Sex Offenders: Identification, Risk Assessment, Treatment and Legal Issues (eds FM Saleh, AJ Grudzinskas, JM Bradford, et al): 101-18. Oxford University Press.

Andrews DA, Bonta J (2007) The Psychology of Criminal Conduct (4th edn). Anderson.

Banse R, Schmidt AF, Clarbour J (2010) indirect measures of sexual interest in child sex offenders: a multimethod approach. Criminal Justice and Behavior 3: 319-35.

Barbaree H, Langton C, Peacock E (2006) Sexual offender treatment for psychopaths: is it harmful? In Sexual Offender Treatment: Controversial Issues (eds WL Marshall, YM Fernandez, LE Marshall, et al): 159-71. John Wiley \& Sons

Beggs SM, Grace RC (2011) Treatment gain for sexual offenders against children predicts reduced recidivism: a comparative validity study. Journal of Consulting and Clinical Psychology 79: 182-92.

Briken P, Hill A, Berner W (2003) Pharmacotherapy of paraphilias with long-acting agonists of luteinizing hormone-releasing hormone: a systematic review. Journal of Clinical Psychiatry 64: 890-7.

Brockman B, Bluglass R (1997) A general psychiatric approach to sexual deviations. In Sexual Deviation (3rd edn) (ed I Rosen): 1-42. Oxford University Press.

Button DM, DeMichele M, Payne BK (2009) Using electronic monitoring to monitor sex offenders. Criminal Justice Policy Review 20: 414-36.

Council of State Governments (2010) Sex Offender Management Policy in the States: Strengthening Policy \& Practice. Final Report. Council of State Governments.

Craig LA, Beech AR (2009) Psychometric assessment of sexual deviance. In Assessment and Treatment of Sex Offenders (eds AR Beech, LA Craig, KD Browne): 89-107. Wiley-Blackwell.

Craig LA, Lindsay WR, Browne KD (2010) Assessment and Treatment of Sexual Offenders with Intellectual Disabilities: A Handbook. WileyBlackwell.

Daffern M, Jones I, Shine J (2010) Offence Paralleling Behaviour. A Case Formulation Approach to Offender Assessment and Intervention. John Wiley \& Sons.

Darjee R, Russell K (2011) The assessment and sentencing of high risk offenders in Scotland: a forensic clinical perspective. In Dangerous People: Policy, Prediction and Practice (eds B McSherry, P Keyzer): 217-32. Routledge.

Darjee R, Russell K (2012) What clinicians need to know before assessing risk in sexual offenders. Advances in Psychiatric Treatment 18: 467-78.

Dowsett J, Craissati J (2008) Managing Personality Disordered Offenders in the Community. A Psychological Approach. Routledge.

Federoff JP, Kuban M, Bradford JM (2009) Laboratory measurement of penile response in the assessment of sexual interests. In Sex Offenders: Identification, Risk Assessment, Treatment and Legal Issues (eds FM Saleh, AJ Grudzinskas, JM Bradford, et al): 89-100. Oxford University Press.

Gannon TA, Cortoni F (2010) Female Sexual Offenders: Theory, Assessment and Treatment. Wiley-Blackwell.

Glasgow DV (2009) Affinity: the development of a self-report assessment of paedophile sexual interest incorporating a viewing time validity measure. In Cognitive Approaches to the Assessment of Sexual Interest in Sexual Offenders (eds. D Thornton, DR Laws): 59-84. Wiley-Blackwell.
Grubin D, Wilcox D, Madsen L (2007) The Use of the Polygraph in Assessing, Treating and Supervising Sex Offenders: A Practitioner's Guide. John Wiley \& Sons.

Grubin D (2008) Medical models and interventions in sexual deviance. In Sexual Deviance: Theory, Assessment and Treatment (2nd edn) (eds DR Laws, WT O'Donoue): 594-610. Guilford Press.

Hanson RK, Morton-Bourgon KE (2005) The characteristics of persistent sexual offenders: a meta-analysis of recidivism studies. Journal of Consulting and Clinical Psychology 73: 1154-63.

Hanson RK, Harris AJR, Scott T, et al (2007) Assessing the Risk of Sexual Offenders on Community Supervision: The Dynamic Supervision Project. User report 2007-05. Department of the Solicitor General of Canada.

Hanson RK, Bourgon G, Helmus L, et al (2009) The principles of effective correctional treatment also apply to sexual offenders: a meta-analysis. Criminal Justice and Behavior 36: 865-91.

Hare RD (2004) The Hare Psychopathy Checklist-Revised Manual (2nd edn). Multi-Health Systems

Hart SD, Cox DN, Hare RD (1995) Manual for Psychopathy Checklist: Screening Version (PCL:SV). Multi-Health Systems.

Hart SD, Kropp PR, Laws DR, et al (2003) Risk for Sexual Violence Protocol (RSVP). Mental Health, Law and Policy Institute, Simon Fraser University.

Hart SD, Logan C (2011) Formulation of violence risk using evidencebased assessments: the structured professional judgment approach. In Forensic Case Formulation (eds P Sturney, M McMurran): 83-106. WileyBlackwell.

Harvey S, Philpot T, Wilson C (2011) A Community-Based Approach to the Reduction of Sexual Reoffending: Circles of Support and Accountability. Jessica Kingsley.

Kemshall H, Wood J (2009) Community strategies for managing highrisk offenders: the contribution of multi-agency public protection arrangements. In Assessment and Treatment of Sex Offenders (eds AR Beech, LA Craig, KD Browne): 535-50. Wiley-Blackwell.

Kingston DA, Yates PM (2008) Sexual sadism - assessment and treatment. In Sexual Deviance: Theory, Assessment and Treatment (2nd edn) (eds DR Laws, WT O’Donohue): 231-49. Guilford Press.

Kropp PR, Hart SD, Webster CD, et al (1995) Manual for the Spousal Assault Risk Assessment Guide (2nd edn). British Columbia Institute Against Family Violence.

Laws DR, Ward T (2011) Desistance from Sex Offending: Alternatives to Throwing away the Keys. Guilford Press.

Letourneau EJ (2002) A comparison of objective measures of sexual arousal and interests: visual reaction time and penile plethysmography. Sexual Abuse: A Journal of Research and Treatment 14: 207-23.

Levenson JS, Hern AL (2007) Sex offender residence restrictions: unintended consequences and community re-entry. Justice Research and Policy 9: 59-73.

Loranger AW (1999) International Personality Disorder Examination Manual. Psychological Assessment Resources.

Marshall WL, Hucker SJ (2006a) Issues in the diagnosis of sexual sadism. Sexual Offender Treatment 1(2) (http://sexual-offender-treatment.org/40. html).

Marshall WL, Marshall LE, Serran GA, et al (2006b) Treating Sexual Offenders: An Integrated Approach. Routledge.

Millon T, Davis R, Millon C (1997) Manual for the MCMI-III. National Computer Systems.

Nichols HR, Molinder I (1984) Multiphasic Sex Inventory. Nichols and Molinder.

Nitschke J, Osterheider M, Mokros A (2009) A cumulative scale of severe sexual sadism. Sexual Abuse: A Journal of Research and Treatment 3 : 262-78.

Rice ME, Harris GT (2003) The size and sign of treatment effects in sex offender therapy. Annals of the New York Academy of Science 989 $428-40$
MCQ answers

1 e $\quad 2$ b $\quad 3 d \quad 4$ b $\quad 5 d$ 
Rich P (2009) Understanding the complexities and needs of adolescent sex offenders. In Assessment and Treatment of Sex Offenders (eds AR Beech, LA Craig, KD Browne): 431-52. Wiley-Blackwell.

Russell K, Darjee R (2012) Managing the risk posed by personality disordered sex offenders in the community: a model for providing structured clinical guidance to support criminal justice services. In Managing Clinical Risk: A Guide to Effective Practice (eds L Johnstone, C Logan): 217-32. Willan Publishing.

Seto MC (2008) Pedophilia and Sexual Offending Against Children: Theory, Assessment and Intervention. American Psychological Association.

Skeem J, Polascheck D, Manchak S (2009) Appropriate treatment works, but how? Rehabilitating general, psychopathic, and high risk offenders. In Psychological Science in the Courtroom: Consensus and Controversies (eds LJ Skeem, KS Douglas, SL Lilienfeld): 358-84. Guilford Press.
Thornton D (2010) Scoring Guide for Risk Matrix 2000: 10/SVC. David Thornton.

de Vogel V, de Ruiter C, Bouman Y, et al (2009) SAPROF. Guidelines for the Assessment of Protective Factors for Violence Risk. English Version. Forum Educatief.

Ward T, Vess J, Collie RM, et al (2006) Risk management or goods promotion: the relationship between approach and avoidance goals in treatment for sex offenders. Aggression and Violent Behaviour 11: 378-93.

Webster CD, Douglas KS, Eaves D, et al (1997) HCR-20. Assessing Risk for Violence. Version 2. Mental Health Law and Policy Institute, Simon Fraser University.

Widiger TA, Huprich S, Clarkin J (2011) Proposals for DSM-5: introduction to special section of Journal of Personality Disorders. Journal of Personality Disorders 2: 135.

\section{MCOs}

Select the single best option for each question stem

1 Which of the following is an objective assessment of sexual interests:

a a thorough sexual history

b a self-report sexual interests questionnaire

c noting what magazines a person looks at

d polygraph interviews

e a viewing-time measure.

\section{Assessing personality disorder in sexual} offenders:

$a$ is rarely necessary in difficult cases

b should involve use of structured assessment instruments

c does not require the assessment of psychopathy

$\mathrm{d}$ making a diagnosis is more important than formulation

e understanding personality rarely has implications for management.

\section{Risk formulation does not involve:}

a providing an understanding of the role of risk factors

b analysing offending

c producing narrative scenarios

d categorising the person as high, medium or low risk

e setting out predisposing, precipitating, perpetuating and protective factors.

\section{Risk management should involve:}

a an long, exhaustive list of restrictions and interventions to ensure nothing is left to chance

b a proportionate individualised approach

c group CBT for all sex offenders

d psychological treatment, even though there is little evidence that it works

e monitoring primarily based on whether the offender is a rapist or child molestor.
5 Treatment approaches:

a anti-libidinals should be used for most sex offenders

b medications that may help with sexual regulation include SSRIs, benzodiazepines and lithium

c an important part of psychological treatment is getting the offender to describe their offence in detail

d treatment should ameliorate risk factors and enhance protective factors

e psychological treatment should not be considered in sexual offenders with psychopathy. 